Collection SFN 10 (2010) 589-603

(C) Owned by the authors, published by EDP Sciences, 2010

DOI: $10.1051 / \mathrm{sfn} / 2010015$

\title{
Diffusion inélastique des neutrons sur les aimants moléculaires
}

\author{
G. Chaboussant
}

\author{
Laboratoire Léon Brillouin, CNRS-CEA (CEA Saclay), 91191 Gif-sur-Yvette, France
}

\section{INTRODUCTION}

Les matériaux magnétiques sont le plus souvent des métaux, des oxydes métalliques, des réseaux ioniques, ou des matériaux moléculaires organiques. Chacune de ces grandes familles de matériaux permet l'étude de systèmes magnétiques extrêmement variés que ce soit en considérant la connectivité magnétique, la force et la symétrie des interactions ou la nature des éléments constitutifs. Dans ce cadre, les matériaux moléculaires magnétiques présentent l'avantage de permettre une extraordinaire variété de formes et de topologies. Les aimants moléculaires sont, en effet, des matériaux cristallins composés d'ions magnétiques pouvant former des systèmes de dimensionnalité différentes [68]. La "dimensionnalité magnétique" du matériau est contrôlée par les interaction d'échange entre spins du réseau ou entre "spins collectifs" des agrégats. Selon la dimensionnalité magnétique, on a alors affaire à une physique à 3D (approches perturbatives ou "de champ moyen", ordre à longue distance), à 2D (supraconducteurs haut-Tc, certains manganites à effet GMR) ou 1D (chaînes et échelles de spins [48]) ou, donc, OD (agrégats, molécules).

A 1D, en particulier, le confinement topologique est très marqué, amenant à des comportements totalement nouveaux par le biais d'un processus de domination-renforcement des fluctuations quantiques déstabilisant les états fondamentaux "classiques"1 comme l'état de Néel dans les antiferromagnétiques (cf théorème de Mermin-Wagner [80]) ou qui conduisent à des états qualitativement différents selon la valeur du $\operatorname{spin}^{2}$ (Haldane [62]). La problématique est quasi-identique ${ }^{3}$ dans les “échelles de spins" (chaînes couplées) $[8,47,48,72]$ ou les chaînes de spin-1/2 dimérisées (échanges alternés permanents ou dimérisation en présence d'une transition spin-Peierls). L'application d'un paramètre extérieur (champ magnétique, pression) permet de sonder, dans tous ces systèmes, les phénomènes de transitions de phase quantique (i.e. à $\mathrm{T}=0$ ) entre un état singulet non magnétique et un état triplet magnétique voire un état ordonné à longue distance [35,36,128].

\footnotetext{
${ }^{1}$ Ceci n'est évidemment que théorique car, dans la plupart des matériaux de basse dimensionnalité magnétique, il peut toujours exister des couplages inter-chaînes ou inter-plans qui induiront à très basse température un ordre à longue distance.

${ }^{2}$ Les chaînes de spin entier ont un état fondamental singulet $(S=0)$ séparé des états excités triplet $(S=1)$ par un gap d'énergie $\Delta$, alors que les chaînes de spin demi-entier n'ont pas de gap dans leur spectre d'excitations. De ce fait, contrairement aux chaînes de spins demi-entiers, les fonctions de corrélations à $\mathrm{T}=0$ décroissent exponentiellement avec la distance pour les chaînes de spin entier, indiquant ainsi que l'état fondamental est désordonné, analogue à un "liquide de spins" [1,81].

${ }^{3}$ La chaîne $S=1$ de Haldane, la chaîne dimérisée $S=1 / 2$ et les échelles de spins $S=1 / 2$ (pour $J_{\perp}>0$ ), appartiennent à la même classe [125]. Dans chaque cas, on peut construire un état fondamental composé de liaisons de valence résonnantes auxquelles il faut fournir une énergie thermique ou magnétique pour créer une excitation triplet. L'interprétation en termes de liaisons de valence [5] a permis de mieux comprendre les propriétés non conventionnelles des chaînes de spins isotropes dans la limite quantique et notamment la différence de nature entre les chaînes de spins entiers et de spins demi-entiers proposée par Haldane. L'état fondamental se décrit alors comme un liquide de spins désordonné, singulet et doté de liaisons de valence à courtes portées.

This is an Open Access article distributed under the terms of the Creative Commons Attribution-Noncommercial License 3.0, which permits unrestricted use, distribution, and reproduction in any noncommercial medium, provided the original work is properly cited.
} 
Un autre aspect central est le rôle et l'influence de la frustration magnétique ${ }^{4}$ dans la nature et l'établissement de certains états fondamentaux. Cette frustration géométrique joue un rôle majeur dans les réseaux triangulaires [90,101], Kagome [21,63,74,97] ou Pyrochlore [24,54] et, de façon générale, dans les systèmes de basse dimensionnalité [71,96,124]. La frustration peut ainsi agir dans le cas de dimères antiferromagnétiques (AFM) faiblement couplés. C'est notamment le cas dans la famille des conposés $\mathrm{XCuCl}_{3}\left(\mathrm{X}=\mathrm{K}, \mathrm{Tl}, \mathrm{NH}_{4}\right)[33,99]$, où les dimères de $\mathrm{Cu}^{2+}(S=1 / 2)$ forment des paires de singulet faiblement couplés. Les deux premiers présentent un gap de spin tandis que ce dernier présente un état fondamental magnétique. Un dernier cas très intéressant est celui du modèle de Shastry-Sutherland [107] qui est réalisé dans $\mathrm{SrCu}_{2}\left(\mathrm{BO}_{3}\right)_{2}[66,85,86]$. Ce composé est un AFM 2D ayant un gap d'énergie au-dessus de l'état fondamental singulet. A basse température, sa courbe d'aimantation présente des plateaux d'aimantation aux valeurs rationnelles 1/8, 1/4 et 1/3 de l'aimantation à saturation et les excitations de spins ne présentent pas de dispersion majeure en raison des effets de frustration qui seuls peuvent rendre compte des plateaux d'aimantation. L'une des caractéristiques des systèmes frustrés est souvent la grande dégénérescence de l'état fondamental; il en résulte que de très faibles perturbations (fluctuations quantiques) ou irrégularités structurelles (défauts, encombrement stérique) peuvent suffire à lever, partiellement ou complètement cette dégénérescence. Cela conduit ainsi à une stabilisation d'un état fondamental particulier parmi l'ensemble des états initialement de même énergie (processus “d'ordre par le désordre" [116] par lequel les fluctuations thermiques levant partiellement la dégénérescence, “ordonnent” pour partie l'état fondamental).

La physique des agrégats moléculaires a pris une grande ampleur ces 15 dernières années en raison de deux facteurs principaux :

- l'existence de très nombreuses formes d'agrégats moléculaires [12,16,44,58,79,88], tant du point de vue de la nucléarité que des couplages d'échange internes ou des propriétés d'anisotropied ;

- La possibilité offerte par les agrégats moléculaires d'étudier le comportement quantique à l'échelle moléculaire ou nanométrique, notamment l'effet tunnel quantique de l'aimantation [50, 103, 113,123].

Les principaux paramètres qui entrent en jeu dans la physique des agrégats sont :

- la nucléarité5,

- la nature des liaisons chimiques assurant les couplages d'échanges,

- l'anisotropie magnéto-cristalline,

- les interactions intermoléculaires qui pilotent la transition vers un ordre 3D éventuel ${ }^{6}$.

Pour les agrégats moléculaires de spin total $S$ et d'anisotropie uniaxiale $D$ négative, la barrière d'énergie $\Delta$ est donnée par $\Delta \approx D S^{2}$ (voir Figure 1). Cette barrière s'oppose à basse température au retournement de l'aimantation et conduit au phénomène de relaxation activée thermiquement. L'objectif de nombreux groupes a donc été d'augmenter la valeur de $\Delta$ permettant de bloquer l'aimantation à des températures les plus élevées possibles ${ }^{7}$. C'est la maximisation du produit $D S^{2}$ qui pilotera l'intérêt technologique

\footnotetext{
4 On parle de frustration magnétique lorsque l'ensemble des couplages d'échange ne peuvent pas être simultanément satisfaits [115] ; l'état fondamental est alors décrit par une superposition d'états quantiques modulés par leur probabilité d'occurrence. C'est le cas par exemple du triangle antiferromagnétique pour lequel seuls deux des trois "liens" d'échange sont satisfaits. Le troisième est indéterminé et l'état fondamental, dans le cas de spin demi-entiers, est doublement dégénéré.

${ }^{5}$ La nucléarité s'entend comme le nombre d'ions magnétiques contenus dans la cellule élémentaire ou dans une molécule. Les propriétés intrinsèques comme la valeur du spin $S$ des ions, leurs connectivités et nucléarités y sont parfaitement contrôlées.

${ }^{6} \mathrm{Si}$ les interactions dipolaires à longue portée sont suffisamment faibles, on peut observer le comportement d'un seul de ces agrégats moléculaires magnétique indépendamment des molécules voisines.

${ }^{7}$ Les stratégies de synthèse consistent à fournir des degrés de liberté de coordination mais aussi des éléments limitateurs qui vont "borner" les possibilités de réaction dans des limites raisonnables et souhaitées. Le choix du ligand, du sel métallique, du pH, de la température et du solvant, des conditions d'oxydo-réduction : tout cela va contribuer au résultat final. L'effort de synthèse s'oriente vers l'utilisation de structures de faible nucléarité qui sont ensuite assemblées ("self-assembly") dans le processus de réaction chimique afin de former des structures en agrégats plus importants ou des structures à dimensionnalité réduite. Cette
} 

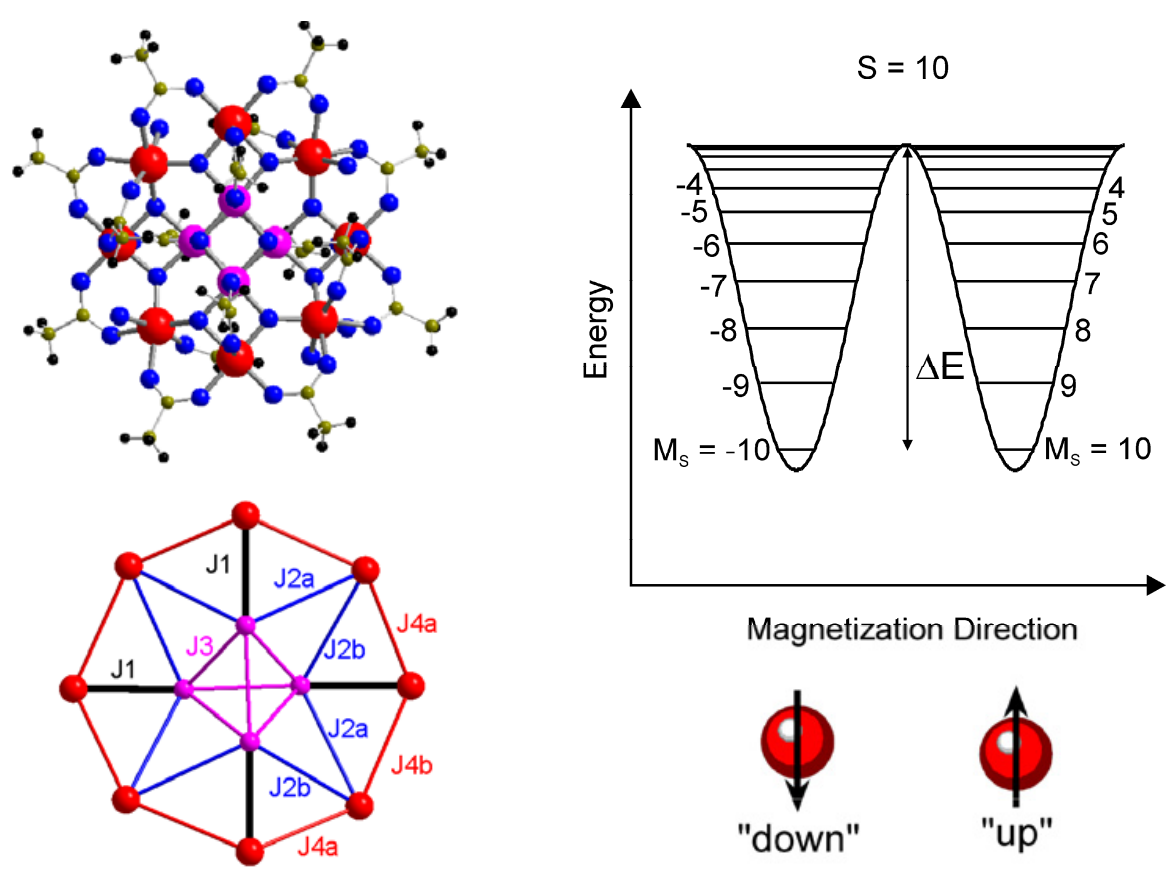

Figure 1. En haut à gauche, structure moléculaire de $\mathrm{Mn}_{12}$-acétate [76], le prototype des aimants moléculaires dits "single-molecule magnets". La molécule est représentée selon l'axe $c$ de facile aimantation. Les sphères solides sont les ions $\mathrm{Mn}^{3+}$ (couche extérieure) et $\mathrm{Mn}^{4+}$ (coeur). La figure du bas (gauche) représente la topologie des couplages d'échanges. La figure de droite montre la barrière d'énergie $\Delta \approx D S^{2}$ de l'état fondamental $S=10$ de cette molécule créee par l'anisotropie uniaxiale $D$ [37].

de ces "aimants à une molécule" ou "single-molecule magnets" (SMM). Dans le cas idéal, dans un régime $T \ll \Delta$, un tel object magnétique présentant une bistabilité pourrait permettre le contrôle de l'état d'aimantation sans dégradation ou perte d'information.

Construire une molécule la plus grande possible, c'est donc se donner la possibilité d'avoir un spin total fondamental $S$ très grand. Evidemment, ce n'est pas souvent le cas car les interactions AFM peuvent contribuer à abaisser le spin total $S$. D'autre part, l'anisotropie magnéto-cristalline totale de la molécule dépend non seulement de la symétrie moléculaire mais aussi des axes locaux d'anisotropie. En d'autres termes, construire une molécule "géante" n'est pas du tout la garantie de construire un système ayant une barrière d'énergie élevée ${ }^{8}$. L'expérience des années récentes semble même faire émerger un concept de "conservation" par lequel l'optimisation d'un des ingrédients permettant d'augmenter $\Delta$ se traduit par la dégradation des autres ingrédients.

Il est certain que le scénario "moléculaire" - en vue d'applications du type "stockage de l'information" - est assez radicalement différent des métaux et oxydes magnétiques formant des nanoobjets de plusieurs milliers d'atomes ou couches minces. La réduction de la taille des particules

stratégie de construction par "briques élémentaires" est une des plus prometteuses, de part la variété des matériaux de départ et de part les nombreuses possibilités de coordination.

8 Par exemple, l'état fondamental le plus élevé a été atteint par A.M. Ako et al. [3] sur le composé $\mathrm{Mn}_{19}\left(\right.$ comprenant $7 \mathrm{Mn}^{2+}$ et $12 \mathrm{Mn}^{3+}$ ) qui possède un état fondamental $S=83 / 2$ mais une anisotropie bien trop faible. En parallèle, la barrière d'énergie la plus élévée a été atteinte par C.J. Milios et al. [82,83] sur un composé de type $\mathrm{Mn}_{6}$ comprenant $6 \mathrm{Mn}^{3+}$. L'état fondamental est $S=12$ et il y a une forte anisotropie uniaxiale $(D=-0.62 \mathrm{~K})$, ce qui donne lieu à une barrière d'énergie de l'ordre de $86 \mathrm{~K}$. 
magnétiques présentant une anisotropie magnéto-cristalline entraîne la formation de monodomaines; la formation de domaines magnétiques n'est plus énergétiquement favorable. Dans le cas uniaxial, une particule de volume $V$ et d'anisotropie $K$, présentera une barrière d'énergie $\Delta=K V$ entre les deux configurations stables du système $(\vec{M}= \pm M \vec{z})$. Si la barrière d'énergie est comparable à $k_{B} T$, la particule perd toute aimantation et entre dans le régime dit "superparamagnétique". La relaxation de l'aimantation, $M(t) \approx M(0)$. $\exp (-t / \tau)$ obéit à la loi d'Arrhenius, $\tau=\tau_{0} \exp \left(\Delta / k_{B} T\right)$. Si on arrive à bloquer l'aimantation dans l'un ou l'autre des puits de potentiel de la barrière, on obtient un objet capable de stocker une unité d'information. Le superparamagnétisme est alors évidemment un obstacle qu'il faut surmonter. La solution réside donc dans l'optimisation soit de la taille des particules $(V)$, aux dépens de la densité, soit en augmentant l'anisotropie $K$ du système. Les tailles caractéristiques nécessaires à l'observation du superparamagnétisme sont de l'ordre de 2 à $50 \mathrm{~nm}$ selon le type de matériaux. Avec les agrégats moléculaires, on cherche à créer une quantité élémentaire ("bit") d'information aussi petite que possible. Ces bits quantiques magnétiques [31,65,75,112] sont l'étape ultime de la miniaturisation, cependant, l'environnement cristallin (interactions avec le monde extérieur), modifiant la stabilité et la dynamique de ces sytèmes (phénomènes de décohérence quantique), conduit à une perte de l'information portée par la molécule et constitue un obstacle majeur au développement de la spintronique moléculaire [94]. Un travail récent de "fonctionalisation" de ces matériaux moléculaires a été entrepris notamment sur des surfaces [34,70,100], ou par organisation en films de LangmuirBlodgett [45,46,102]. En parallèle les aimants moléculaires sont des systèmes modèles pour l'étude de certaines fonctions biologiques (transport d'oxygène [19,25,56,93], activité enzymatique [43,126, 127]). Le champ d'intervention des agrégats moléculaires est donc large : de systèmes modèles pour le magnétisme quantique ou certaines fonctions biologiques aux développements de l'électronique moléculaire.

\section{DIFFUSION DES NEUTRONS APPLIQUÉE AUX AIMANTS MOLÉCULAIRES}

Comme dans l'ensemble des systèmes magnétiques de basse dimensionnalité ou frustrés, la diffusion inélastique des neutrons ${ }^{9}$ joue un rôle important pour comprendre les agrégats magnétiques moléculaires [12,22]. On citera la détermination des paramètres d'anisotropie de $\mathrm{Mn}_{12}$ [14,84, 120], de $\mathrm{Fe}_{8}$ [4,29], $\mathrm{Ni}_{4}$ [108], $\mathrm{Mn}_{4} \mathrm{X}$ [6], $\mathrm{Mn}_{9}$ [15], $\mathrm{Cr}_{8}$ [30], etc. Grâce aux spectromètres à très haute résolution [14] il est possible de déterminer avec une très grande précision les termes d'anisotropie d'ordre supérieur dans $\mathrm{Mn}_{12}$-acétate qui sont responsables de l'effet tunnel macroscopique. Les mesures sous pression hydrostatique de $\mathrm{Mn}_{12}$ et $\mathrm{Mn}_{4}$ [108,109] ont permis d'entrevoir les mécanismes microscopiques qui pilotent l'anisotropie. La diffusion des neutrons est également largement utilisée pour déterminer les interactions d'échange à l'intérieur des agrégats, celle-ci étant très supérieure aux mesures de susceptibilité - qui n'est sensible qu'à la somme pondérée des couplages d'échange - quand des effets de frustration géométrique sont à l'oeuvre [12,13,37,39]. C'est notamment le cas dans trois exemples qui sont discutés par la suite: $\mathrm{Mn}_{4}$-butterfly, $\mathrm{Mn}_{12}$-acétate et $\mathrm{V}_{15}$.

\subsection{Frustration magnétique dans $\mathrm{Mn}_{4}$ butterfly et $\mathrm{V}_{15}$}

Le composé appelé $\mathrm{Mn}_{4}$-butterfly ${ }^{10}$ est exemplaire de l'effet de la frustration magnétique "intra-agrégat" dans un système "simple" : il consiste en 4 ions $\mathrm{Mn}^{3+}(S=2)$ disposés selon l'arrangement géométrique

\footnotetext{
${ }^{9}$ Les détails concernant la section efficace de diffusion des neutrons ne seront pas explicités dans cet article. Le lecteur est invité à se reporter aux articles précédents (S. Raymond, H. Schober et F. Moussa) et pourra approfondir le sujet en consultant les ouvrages de S. Lovesey [77] et G.L. Squires [110] ou, plus spécifiquement, les publications de A. Furrer-H.U. Güdel [51,52,60] et d'O. Waldmann et al. $[117,119,121]$.

$10\left[\mathrm{Mn}_{4} \mathrm{O}_{2}\left(\mathrm{O}_{2} \mathrm{CPh}\right)_{6}(\mathrm{dpm})_{2}\right]:$ cf $[23]$.
} 

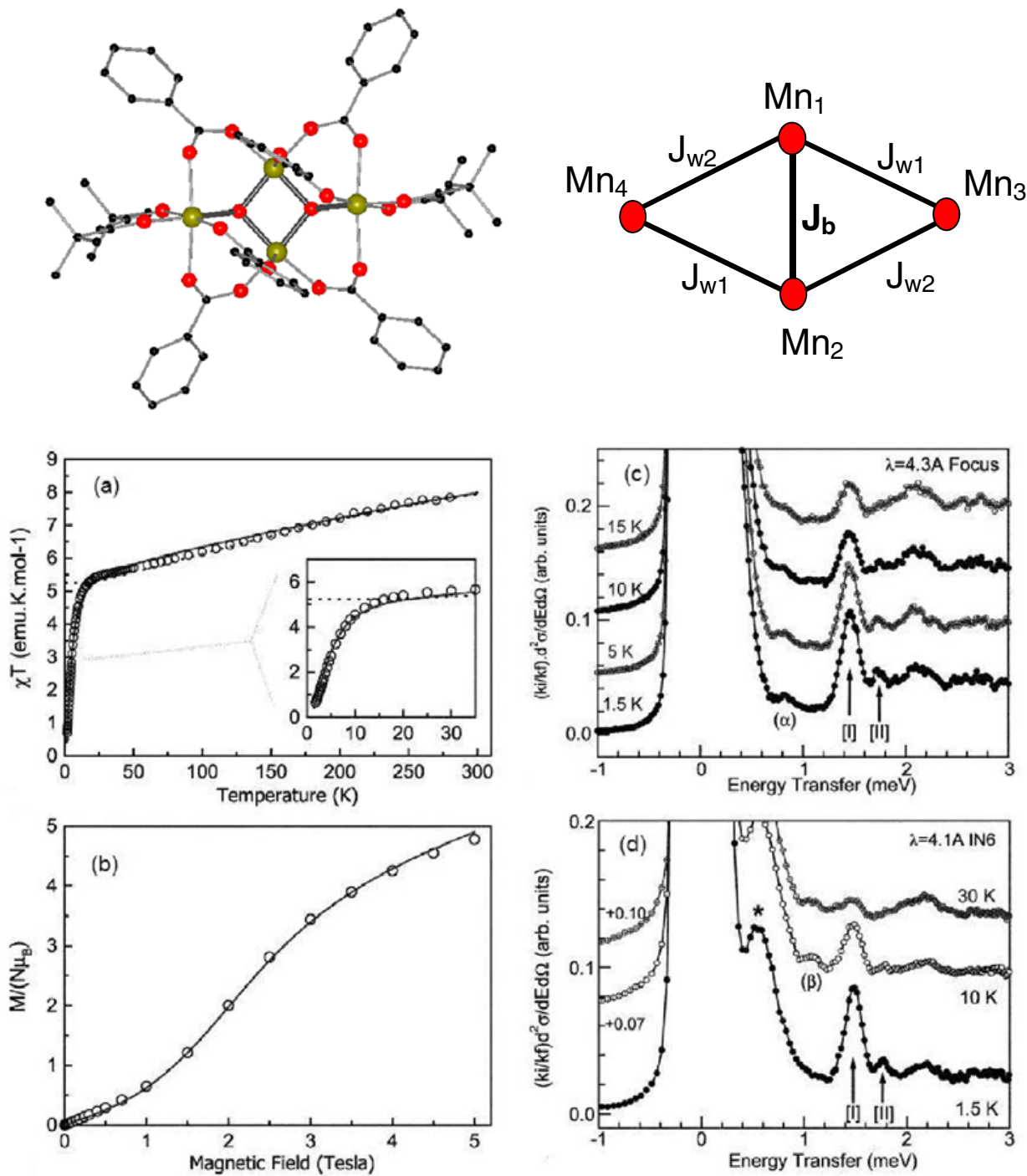

Figure 2. En haut : Molécule et schéma des échanges: $\mathrm{Mn}_{4}$-butterfly. Structure de $\left[\mathrm{Mn}_{4} \mathrm{O}_{2}\left(\mathrm{O}_{2} \mathrm{CPh}\right)_{6}(\mathrm{dpm})_{2}\right]$ vue le long de l'axe $\vec{c}$. Les sphères solides symbolisent les ions $\mathrm{Mn}^{3+}$ ions. Les ions $\mathrm{Mn}^{3+}$ extérieurs ont un environnement octahèdrique distordu. Représentation schématique de l'agrégat et de couplages d'échanges. En bas à gauche (a-b) : aimantation et susceptibilité SQUID obtenues sur $\mathrm{Mn}_{4}$-butterfly. Les courbes continues représentent un ajustement des paramètres (voir texte). En bas à droite (c-d): Spectres INS à $\lambda=4.3 \AA$ obtenu sur FOCUS à différentes températures. Les spectres représentent la somme intégrée sur tous les détecteurs $\left(10^{\circ}<2 \theta<130^{\circ}\right)$. (b) Spectre INS à $\lambda=4.3 \AA$ obtenu sur IN6 à différentes températures. Les spectres représentent la somme intégrée sur les détecteurs aux petits angles (angle moyen $2 \theta=40.4^{\circ}$ ); La gamme de $Q$ est $Q \approx 1.0-1.5 \AA^{-1}$.

dit "butterfly", où deux Mn forment le "corps" et deux autres les "ailes" de part et d'autre. Les mesures de diffusion inélastique des neutrons [13], couplées aux mesures magnétométriques, ont montré que le couplage $J_{b}=-4.8 \mathrm{meV}$ entre les deux spins de coeur $\mathrm{Mn}_{1}$ et $\mathrm{Mn}_{2}$ (cf Fig. 2) est dominant et antiferromagnétique, tandis que les couplages $J_{w 1}=J_{w 2}= \pm 0.1 \mathrm{meV}$ entre les atomes centraux et les atomes extérieurs, $\mathrm{Mn}_{3}$ et $\mathrm{Mn}_{4}$, sont beaucoup plus faibles. Dans cette situation, le dimère 

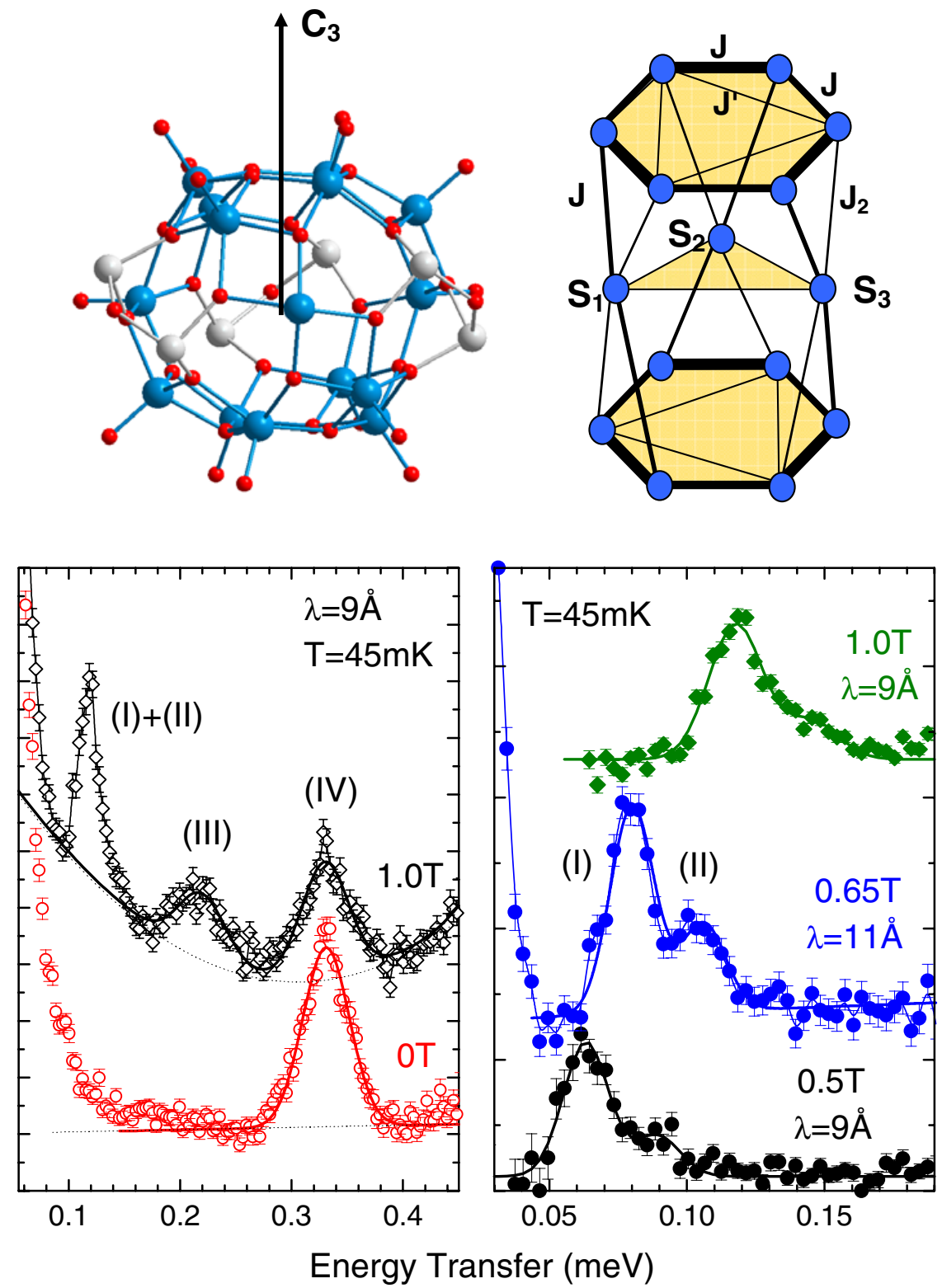

Figure 3. En haut à gauche: Structure de l'anion dans $\left[\mathrm{V}_{15} \mathrm{As}_{6} \mathrm{O}_{42}\right]^{6-}$. Les $15 \mathrm{~V}^{4+}$ forment deux couches hexagonales distordues extérieures et un triangle interne. Schéma de droite: Représentation schématique des couplages d'échange entre les ions $\mathrm{V}^{4+}$ en considération des types de ponts oxygène présents dans les chemins d'échange (distance et angle). En bas: Spectres inélastiques à $45 \mathrm{mK}$ (IN5) des neutrons de $\mathrm{V}_{15}$ pour différentes valeurs de champ magnétique et de longueur d'ondes (voir texte et Ref. [39]).

central est dans un état singulet $(S=0)$ à basse température et les ions extérieurs sont très largement découplés. L'état fondamental, dans cette hypothèse, est donc dégénéré 5 fois. Il a été toutefois montré que l'introduction d'une anisotropie uniaxiale $\left(D_{i}\right)$ sur les sites octahédriques $\mathrm{Mn}_{3}$ and $\mathrm{Mn}_{4}$ et des couplages $J_{w 1}$ et $J_{w 2}$ différents est nécessaire pour expliquer le comportement à basse température 

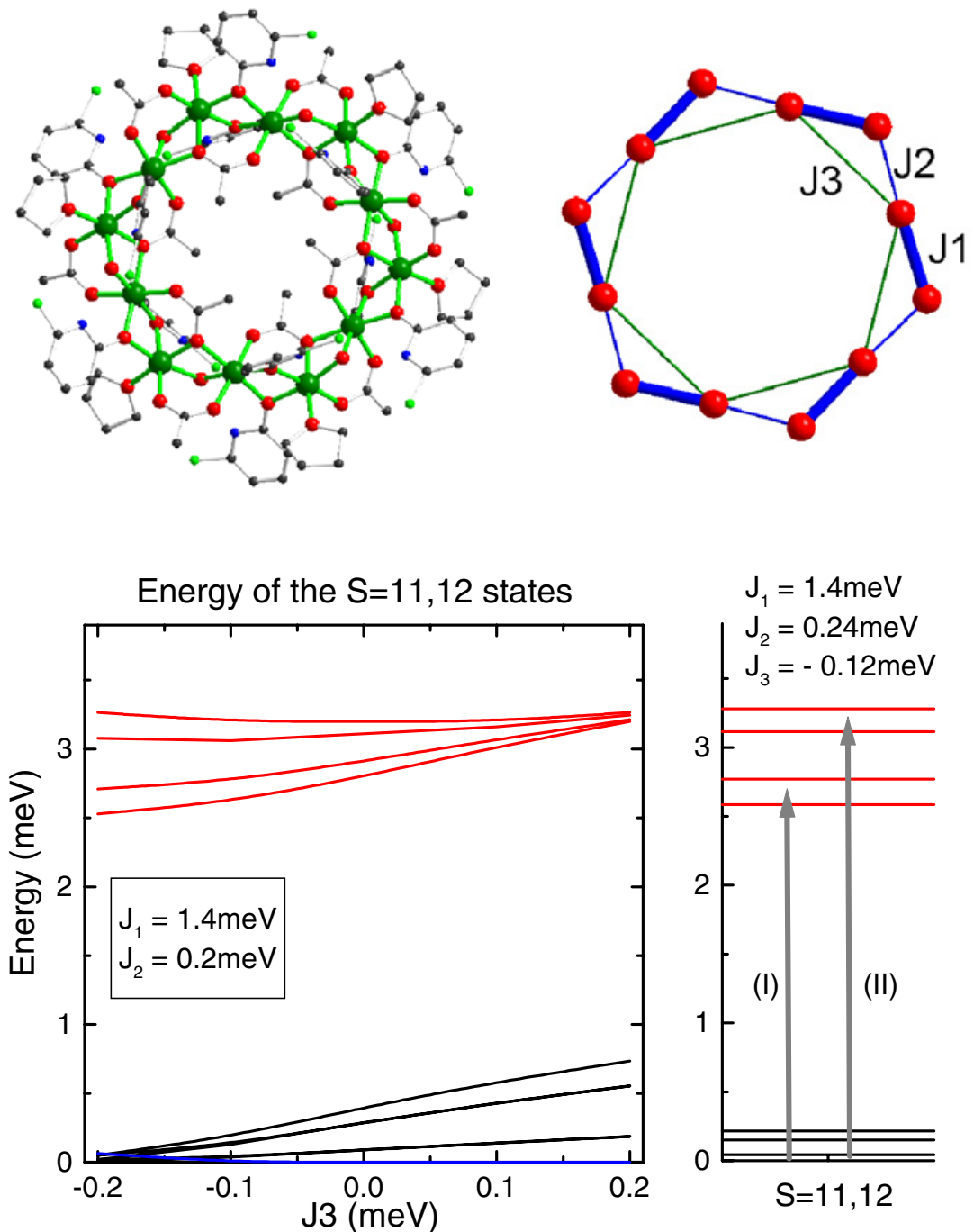

Figure 4. En haut : Structure de $\mathrm{Ni}_{12}$ et représentation des couplages d'échange. En bas: Evolution des niveaux d'énergie en fonction de l'interaction de deuxième voisin. A gauche sont représentés les niveaux d'énergie calculés pour les états $S=11$ et $S=12$ en fonction de l'interaction de second voisin $J_{3}$ tandis qu'à droite est tracé le diagramme des niveaux d'énergie pour $J_{1}=1.40, J_{2}=0.24$ et $J_{3}=-0.12 \mathrm{meV}$. Les deux flèches indiquent la position des transitions observées.

(cf Fig. 2). L'effet principal de l'anisotropie est de lever la quintuple dégénérescence de l'état fondamental mais on voit aussi que la frustration intrinsèque générée par la structure "butterfly" [67] est levée par la présence de couplages "body-wing" légèrement différents les uns des autres et par l'anisotropie magnéto-cristalline. Les effets de frustration conduisent à des propriétés magnétiques qui n'auraient pas pu être comprises sans l'apport des neutrons car la détermination univoque de tous les paramètres pertinents sur la seule base des mesures de susceptibilité n'est pas possible [78].

Un autre cas de frustration est le polyoxovanadate $\mathrm{K}_{6}\left[\mathrm{~V}_{15} \mathrm{As}_{6} \mathrm{O}_{42}\left(\mathrm{D}_{2} \mathrm{O}\right)\right] \cdot 8 \mathrm{D}_{2} \mathrm{O}\left(\mathrm{V}_{15}\right)$ qui contient $15 \mathrm{~V}^{4+}$ spins $(S=1 / 2)$ distribués sur 2 hexagones extérieurs et un triangle central (voir Figure 3 ) $[10,55,87]$. La structure des couplages d'échange entre ces trois "couches" conduisent à un modèle 

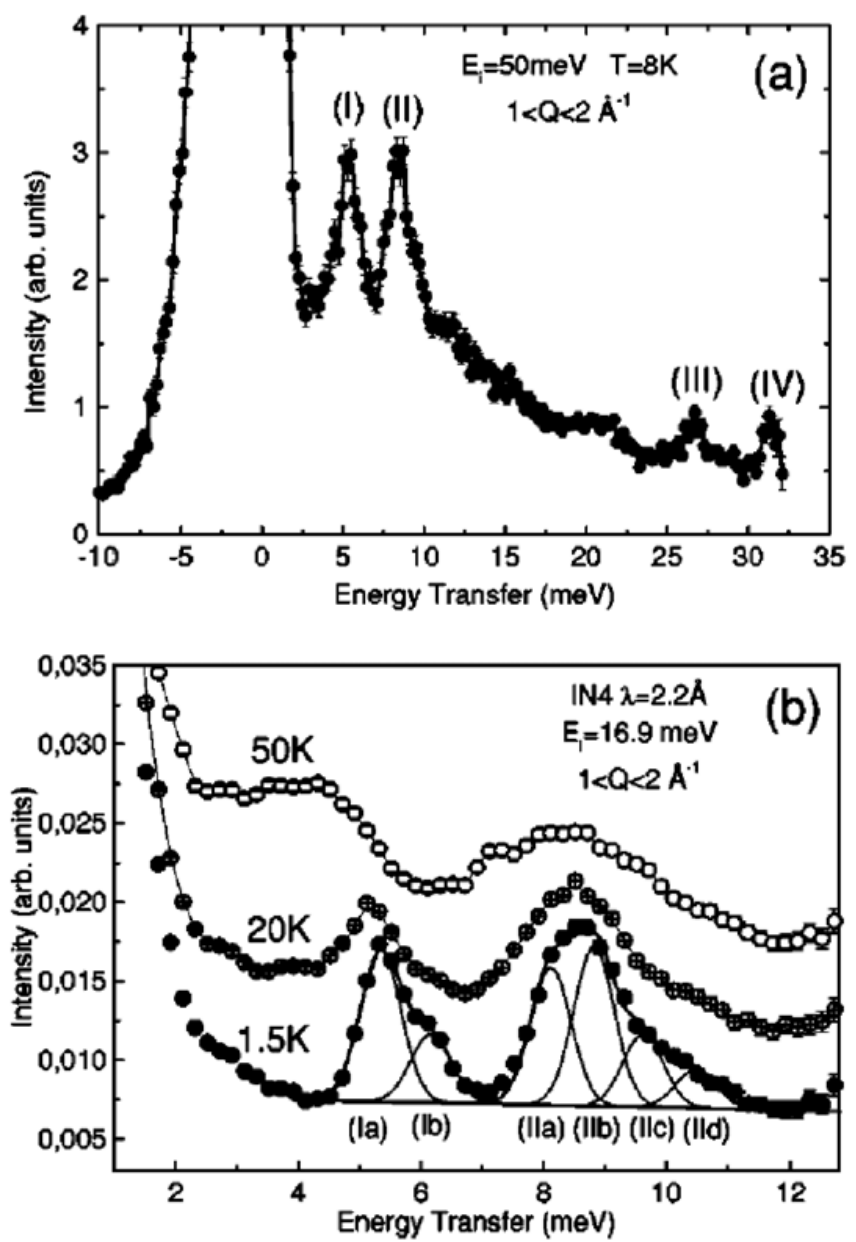

Figure 5. (a) Spectre INS obtenu sur MARI à $\mathrm{T}=8 \mathrm{~K}$ et $E_{i}=50 \mathrm{meV}$. La gamme en $Q$ est restreinte à $1 \leq Q$ $\leq 3 \AA^{-1}$. (b) Spectre INS obtenu sur IN4 à $E_{i}=16.9 \mathrm{meV}$ pour différentes températures. La gamme en $Q$ est restreinte à $1 \leq Q \leq 2 \AA^{-1}$.

effectif des propriétés magnétiques qui se limite à un trimère antiferromagnétique $(J \approx 0.315 \mathrm{meV})$ de spin-1/2 conduisant à un état fondamental composé de deux doublets de Kramers $S=1 / 2$ (dégénérés en énergie) et un état excité quartet $S=3 / 2[38,39]$. Les mesures de diffusion des neutrons ont mis en évidence la présence d'un gap d'énergie $\left(2 \Delta_{0} \approx 27 \mu \mathrm{eV}\right)$ entre les deux doublets de Kramers ce qui fait de $\mathrm{V}_{15}$ un système prototype pour l'étude des systèmes à deux niveaux quantiques (modèle de Landau-Zener) et, notamment, les effets de relaxation (adiabatique) et de couplages avec les phonons (effet Bottleneck) [40,41,49]. Les spectres inélastiques obtenus à $\lambda=7.5$ et 9.0 (voir Figure 3) pour différentes valeurs du champ magnétique sur IN5 ont permis de déterminer les couplages d'échange, la valeur du gap entre les deux doublets et l'origine physique de ce gap (distorsion causée par les molécules d'eau située au centre des cavités sphériques de $\mathrm{V}_{15}$ ou par un désordre (occupation partielle) de l'eau dans le réseau [10,42,55]) par le biais de l'intensité et de la dépendance en $Q$ des transitions [39]. $\mathrm{V}_{15}$ montre que des variations infimes de la structure (un "désordre") relâche une partie de la frustration magnétique en favorisant un état fondamental plutôt qu'un autre (un “ordre”). 

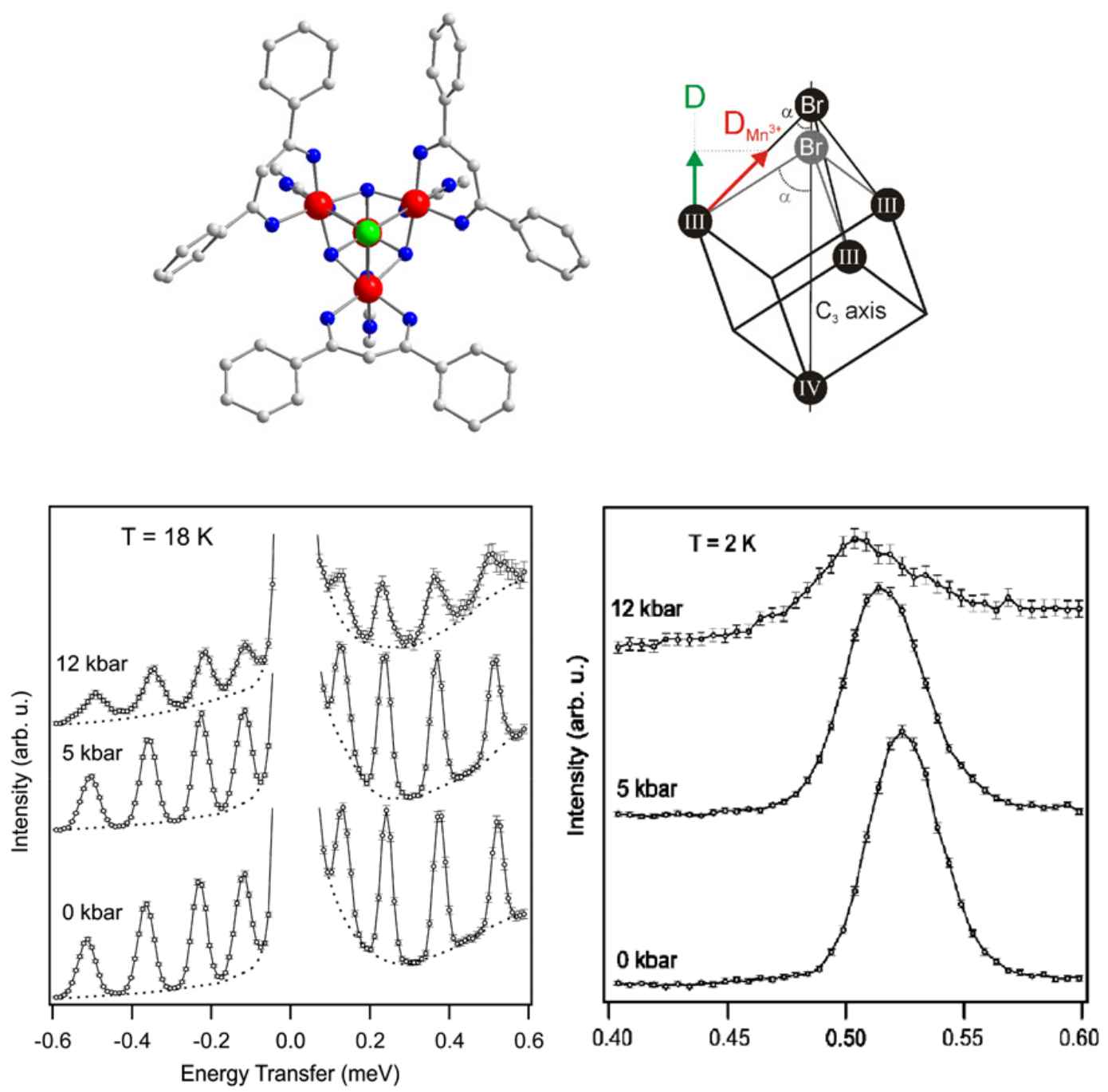

Figure 6. En haut à gauche: Représentation du tétramère cubane $\left[\mathrm{Mn}_{4} \mathrm{O}_{3} \mathrm{Br}(\mathrm{OAc})_{3}(\mathrm{dbm})_{3}\right]$. En bas (gauche): Spectre à $18 \mathrm{~K}$ et $\lambda=8$ Ån fonction de la pression, montrant l'ensemble du spectre inélastique et toutes les transitions $\Delta M= \pm 1$. En bas (droite): Spectres montrant la transition de plus grande énergie $M_{S}=9 / 2 \rightarrow M_{S}=$ $7 / 2$ à différentes pressions appliquées [109].

\subsection{L'anneau de $\operatorname{spin} \mathrm{Ni}_{12}$}

Le complexe cyclique $\mathrm{Ni}_{12}{ }^{11}$ est un anneau de $\operatorname{spin}^{12}$ de 12 atomes $\mathrm{Ni}^{2+}(S=1)$, dans lequel les échanges dominants sont ferromagnétiques et conduisent à un état fondamental $S=12$ (Figure 4) [7]. Une faible anisotropie axiale conduit également à une barrière d'énergie de l'ordre de $10 \mathrm{~K}$. Si l'échange

\footnotetext{
$11\left[\mathrm{Ni}_{12}(\mathrm{chp})_{12}\left(\mathrm{O}_{2} \mathrm{CMe}\right)_{12}(\mathrm{THF})_{6}\left(\mathrm{H}_{2} \mathrm{O}\right)_{6}\right](\mathrm{chp}=$ 6-chloro-2-pyridonate) [17].

12 Les anneaux FM ou AFM [2,27,32,73,104,118] sont considérés comme des modèles en "miniature" des systèmes undimensionnels (chaînes) en raison de leur topologie : cela supposerait que les concepts qui pilotent la physique à $1 \mathrm{D}$ pourraient s'appliquer "tels que" aux anneaux de spins. Cependant, les réalisations expérimentales montrent que les effets de taille finie des anneaux de spins sont trop dominants pour que l'on puisse y observer expérimentalement une véritable physique à 1D. Malgré cela, les anneaux de spins constituent un terrain d'investigation de choix des effets quantiques.
} 
dominant entre les ions $\mathrm{Ni}^{2+}$ est ferromagnétique, le modèle d'une chaîne uniforme $\left(J_{1}=J_{2}\right.$ et $\left.J_{3}=0\right)$ ne s'accorde pas avec les résultats de diffusion des neutrons et il faut introduire une dimérisation de l'échange entre lers voisins ainsi qu'un échange entre deuxième voisin pour obtenir un accord entre les modèles et les mesures obtenues aux neutrons : $J_{1}=1.40(5) \mathrm{meV}, J_{2}=0.24(3) \mathrm{meV}$ et $J_{3}=-0.12(2) \mathrm{meV}$ (Figure 4). Ces couplages d'échange reflètent la présence de trois chemins de super-échanges différents dans la structure, à la fois par les distances et angles de liaisons et par le nombre de chemins possibles entre deux sites voisins de Ni. Les valeurs obtenues et la hiérarchie de ces valeurs est en accord qualitatif avec les études de corrélations magnétostructurales existantes sur les agrégats de Nickel $[61,91]$. Des calculs récents de DFT (Density Functional Theory) et comparaison avec la susceptbilité [28] par Venegas et al. [114] confirment la dimérisation, même si celle-ci apparaît moins marquée qu'à partir des mesures de diffusion des neutrons, mais indiquent que le couplage entre seconds voisins $J_{3}$ devrait être FM tout en restant faible. Cependant, la présence d'un anisotropie unaxiale $D$, faible mais suffisante pour induire une barrière d'énergie de l'ordre de $10 \mathrm{~K}$ et de faibles couplages inter-moléculaires [7,69] suffisent à considérablement modifier la susceptibilité à basse température.

\section{3 $\mathrm{Mn}_{12}$-acétate}

$\mathrm{Mn}_{12}$-acétate ${ }^{13}$ (structure représentée sur la Figure 1) est un composé à valence mixte $\left(\mathrm{Mn}^{3+} / \mathrm{Mn}^{4+}\right)$ où les ions magnétiques sont disposés en deux groupes : un coeur central composé d'un tétrahèdre de $4 \mathrm{Mn}^{4+}$ ions $(S=3 / 2)$ et une couronne extérieure de $8 \mathrm{Mn}^{3+}$ ions $(S=2)[9,26,105,106]$. Dans l'approximation tétragonale, il y a trois sites différents de $\mathrm{Mn}$ et quatre couplages d'échange différents par leur nature et les liaisons impliquées. Les couplages antiferromagnétiques imposent une organisation ferrimagnétique ${ }^{14}$ conduisant à un état fondamental $S=10[105,106]$, confirmé par de nombreuses expériences $[11,53,64,84,113]$. Le terme axial de l'anisotropie magnétocristalline $D \approx-0.65 \mathrm{~K}$, induit principalement par la distorsion Jahn-Teller des octahèdres $\mathrm{MnO}_{6}$ des ions $\mathrm{Mn}^{3+}$ situés sur la couronne, génère une barrière d'énergie $\Delta=|D| S^{2} \approx$ $65 \mathrm{~K}(=5.7 \mathrm{meV})$ entre les états $M_{S}= \pm 10$ et $M_{S}=0$ qui bloque la relaxation thermique de l'aimantation à basse température $[18,59,106,129]$. Pour comprendre réellement la formation de l'état $S=10$ et lever les incertitudes concernant les couplages d'échange [20,92,95,98], l'utilisation de la diffusion des neutrons, combinée aux mesures connues de susceptibilité et d'aimantation, a été utilisée dans une gamme d'énergie suffisamment importante (Figure 1) pour évaluer la position des états excités et, par suite, proposer un ensemble de couplages d'échange satisfaisant les principales mesures expérimentales [37]. La structure de l'état fondamental est à 80-90\% en accord avec l'arrangement ferrimagnétique classique de la Figure 1. L'alignement "classique" se trouve justifié par la prééminence des couplages AFM $J_{1}=67 \pm 3 \mathrm{~K}$ et $J_{2}=62 \pm 3 \mathrm{~K}$ qui forcent l'arrangement anti-parallèle des ions de la couronne et du coeur. Les couplages AFM $J_{3}=8 \pm 3 \mathrm{~K}$ et $J_{4}=6 \pm 3 \mathrm{~K}$ représentent des déviations mineures par rapport à l'état classique mais assurent la stabilisation de l'état fondamental $S=10$ de la molécule de $\mathrm{Mn}_{12}$-acétate. Les premiers états excités $S=9$, qui sont situés à 4-8 $\mathrm{meV}$ au-dessus du fondamental, sont relativement proches du haut de la barrière $\Delta$ du fondamental et vont jouer un rôle dans la dynamique de l'aimantation quand la température sera de l'ordre de grandeur de $\Delta$. Ce résultat n'aurait pas pu être obtenu à partir des mesures standard de magnétométrie en raison de l'amplitude des couplages d'échange (le régime de Curie-Weiss ne peut pas être observé avant que l'échantillon ne se décompose) et de la compétition d'au moins 4 interactions d'échange, ferromagnétique et antiferromagnétique, entre les différents Mn de l'agrégat $\mathrm{Mn}_{12}$. Une combinaison de mesures à basse $(0-2 \mathrm{meV})$ et haute énergie $(2-50 \mathrm{meV})$ confrontée à des simulations numériques permettent d'obtenir une solution unique de couplages d'échange satisfaisant à

$13\left[\mathrm{Mn}_{12} \mathrm{O}_{12}\left(\mathrm{CH}_{3} \mathrm{COO}\right)_{16}\left(\mathrm{H}_{2} \mathrm{O}\right)_{4}\right] \cdot 4 \mathrm{H}_{2} \mathrm{O}_{2} \mathrm{CH}_{3} \mathrm{COOH}$.

14 Les $8 \mathrm{Mn}^{3+}(S=2)$ étant orientés antiparallèlement aux 4 spins $\mathrm{Mn}^{4+}(S=3 / 2)$. 
la fois les données de diffusion des neutrons, de susceptibilité et un état fondamental $S=10$ (voir les spectres de la figure 5).

\subsection{Effets de la pression}

L'utilisation de la pression hydrostatique est une autre voie (analogue en fait à la "pression chimique" produite par exemple par des ligands différents) très prometteuse, permettant de sonder les corrélations entre structure et anisotropie dans les aimants moléculaires. Les premières mesures d'aimantation sous pression d'agrégats moléculaires ont été réalisées récemment et ont montré une augmentation de la barrière d'énergie avec des pressions croissantes [89,111]. Comme dans le cas d'une modification chimique, l'application de la pression conduit à une altération des propriétés d'anisotropie de l'agrégat. La pression réduit les distances entre atomes, donc les couplages électroniques et recouvrements orbitaux s'en trouvent modifiés. Cependant, les conséquences sur les termes d'anisotropie peuvent être très variables car ce sont distances et angles de liaisons qui évoluent avec la pression. Pour illustrer le propos, deux systèmes aux comportements antagonistes sont discutés: $\mathrm{Mn}_{12}$-acétate et $\left[\mathrm{Mn}_{4} \mathrm{O}_{3} \mathrm{Br}(\mathrm{OAc})_{3}(\mathrm{dbm})_{3}\right]$ (dénoté $\mathrm{Mn}_{4} \mathrm{Br}$ ), un composé de structure cubane ayant un état fondamental $S=9 / 2$ [6,109] (voir la figure 6). Dans le cas de $\mathrm{Mn}_{12}$-acétate, le modèle de recouvrement d'orbitale [57] permet d'estimer à $2.2 \%$, en excellent accord avec les résultats expérimentaux, l'évolution en fonction de la pression moyennant quelques hypothèses (même compressibilité des Mn-O). L'analyse indique que le terme déterminant dans l'augmentation de l'anisotropie globale de l'agrégat est l'anisotropie individuelle $\mathrm{D}_{\mathrm{Mn}^{3+}}$. Si la pression a pour effet d'augmenter l'anisotropie, et donc la barrière d'énergie, de $\mathrm{Mn}_{12}$-acétate, la situation s'inverse pour $\mathrm{Mn}_{4} \mathrm{Br}$. Ce composé est un membre de la famille $\left[\mathrm{Mn}_{4} \mathrm{O}_{3} \mathrm{X}(\mathrm{OAc})_{3}(\mathrm{dbm})_{3}\right](\mathrm{dbm}=$ monoanion de dibenzoylmethane $)$ où $\mathrm{X}=\mathrm{Br}, \mathrm{Cl}, \mathrm{OAc}, \mathrm{F}, \mathrm{OSi}\left(\mathrm{CH}_{3}\right)_{3}$. Plusieurs études de diffusion des neutrons ont été faites pour caractériser les paramètres d'anisotropie en fonction de $X[6,122]$. L'agrégat comprend 3 ions $\mathrm{Mn}^{3+}(S=2)$ couplés AFM avec un $\mathrm{Mn}^{4+}$ $(S=3 / 2)$ à l'apex du prisme trigonal. L'arrangement AFM conduit à un état fondamental $S=9 / 2$ et l'anisotropie produit une barrière d'énergie comprise entre 0.935 et $1.31 \mathrm{meV}$ selon la nature de $X$. En étudiant plus particulièrement l'effet de pression sur les composés $\mathrm{Mn}_{4} \mathrm{Br}$ on constate une diminution de l'énergie des transitions et donc une baisse de l'anisotropie axiale de $D=-0.0627(1) \mathrm{meV}$ à pression ambiante jusqu'à $D=-0.0603$ (3) meV à $12 \mathrm{kbars}$ [109] (La figure 6 montre la transition observée à basse température en fonction de la pression). Cette baisse s'explique par une modification des angles de liaison entre $\mathrm{Mn}^{3+}$ et atome $X$ (terme de la forme $3 \cos \alpha_{i}^{2}-1$ ). Des calculs de constante de force montrent que la liaison $\mathrm{Mn}-\mathrm{Br}$ est plus compressible que la liaison $\mathrm{Mn}-\mathrm{O}$, ce qui implique que, sous pression, l'axe d'anisotropie des ions $\mathrm{Mn}^{3+}$ est modifié. Cette torsion des axes Jahn-Teller conduit à une réduction nette de l'anisotropie globale de l'agrégat. Une estimation numérique montre qu'une variation de $0.25^{\circ}$ (de $42.5^{\circ}$ à $42.75^{\circ}$ ) des angles de liaison induit une diminution de l'ordre de $2 \%$ de l'anisotropie globale ce qui est qualitativement en accord avec la variation expérimentale de $3.8 \%$ mesurée par INS.

\section{CONCLUSIONS}

Les travaux effectués sur les agrégats moléculaires magnétiques, notamment par l'utilisation de la diffusion inélastique des neutrons montre la capacité des neutrons à (1) révéler la hiérarchie des échanges magnétiques dans les systèmes multi-échanges en compétitition, (2) mettre à jour les termes d'ordre supérieur de l'anisotropie magnéto-cristalline, au même titre que la RPE mais sur une gamme d'énergie plus élevée, et enfin (3) permettre un accès aux hautes énergies jusqu'à 200 meV en pratique. Au même titre que pour la plupart des systèmes dits "fortement corrélés", la diffusion des neutrons offre ainsi souvent la possibilité de trancher "net" des problématiques théoriques ou liées aux résultats expérimentaux. Les progrès récents en instrumentation neutronique (augmentation des flux, nombre et qualité des détecteurs, environnements échantillon spécialisés, outils informatiques, etc.) ont ouvert de 
nouvelles possibilités d'expérimentation vers des systèmes plus complexes, des volumes d'échantillons plus petits, des mesures en "temps réel", etc.

La physique des agrégats moléculaires continue de se développer et de profiter des progrès réalisés par les chimistes pour concevoir et mettre en oeuvre de nouvelles stratégies de synthèse. Celles-ci ont permis de rendre disponible un nombre très important de structures inédites par leur topologies, nucléarités, structures cristallines. L'incessant va-et-vient entre le physicien et le chimiste, ce travail de concert, a été un élément essentiel du succès de ce genre d'études. Du point de vue des physiciens, la découverte de nouvelles molécules a ouvert la voie vers des études physiques inédites car ces agrégats constituent un mini-laboratoire des effets quantiques à l'échelle moléculaire. Les récentes avancées obtenues dans la synthèse de molécules magnétiques, aux propriétés mieux définies ou potentiellement plus intéressantes, sont le fruit de ces allers et venues entre physiciens et chimistes.

\section{Remerciements}

Je tiens particulièrement à remercier Professeur H-U. Güdel (Université de Berne, Suisse) et l'ensemble des personnes, étudiants et chercheurs, qui ont contribué aux résultats présentés dans cet article. Les travaux présentés dans cet article ont été partiellement financés par la Swiss National Science Foundation (NFP 47) et par les programmes européens TMR Molnanomag (No. HPRN-CT-199900012) et TMR Quemolna MRTN-CT-2003-504880.

\section{Références}

[1] I. Affleck et al, Phys. Rev. Lett. 59, 799 (1987).

[2] M. Affronte et al, Phys. Rev. Lett. 68, 104403 (2003).

[3] A.M. Ako et al, Angew. Chem. Int. Ed. 45, 4926 (2006).

[4] G. Amoretti G, et al, Phys. Rev. B 62, 3022 (2000).

[5] P.W. Anderson, Mat. Res. Bull. 8, 153 (1973); Science 235, 1196 (1987).

[6] H. Andres, et al, J. Am. Chem. Soc. 122, 12469 (2000).

[7] H. Andres, et al, Chem. Eur. J. 21, 4867 (2002).

[8] M. Azuma et al, Phys. Rev. Lett. 73, 3463 (1994).

[9] B. Barbara B, et al, J. Mag. Magn. Mat. 200, 167 (1999).

[10] A.L. Barra, D. Gatteschi, L. Pardi, A. Müller, et J. Döring, J. Am. Chem. Soc. 114, (1992) 8509.

[11] A.L. Barra, D. Gatteschi D, R. Sessoli, Phys. Rev. B 56, 8192 (1997).

[12] R. Basler, et al, Chem. Phys. Chem. 4, 910 (2003).

[13] R. Basler, et al, Polyhedron, 222471 (2003).

[14] R. Bircher, et al, Phys. Rev. B 70, 212413 (2004).

[15] R. Bircher, et al, Polyhedron 24, 2455 (2005).

[16] R. Bircher, et al, Adv. Funct. Mater. 16, 209 (2006).

[17] A.J. Blake et al, J. Soc. Chem. Commun. 2363 (1994).

[18] L. Bokacheva, A.D. Kent, and M.A. Walters, Phys. Rev. Lett. 85, 4803 (2000).

[19] C. Boskovic et al, Inorg. Chem 43, 5053 (2004).

[20] D.W. Boukhvalov, et al, Phys. Rev. B 65, 184435 (2002).

[21] C. Broholm, et al, Phys. Rev. Lett 65, 3173 (1990).

[22] R. Caciuffo, et al, Phys. Rev. B 71, 174407 (2005).

[23] C. Cañada-Vilalta, J.C. Huffman and G. Christou, Polyhedron 20, 1785 (2001).

[24] B. Canals, C. Lacroix, Phys. Rev. Lett. 80, 2933 (1998).

[25] A. Caneschi et al, Inorg. Chem. 34, 4660 (1995).

[26] A. Caneschi, et al, J. Mag. Mag. Mat. 200, 182 (1999).

[27] A. Caneschi et al, Angew. Chem. Int. Ed. 38, 1295 (1999).

[28] C. Cadiou, et al, Chem. Comm. (24): 2666 (2001). 
[29] S. Carretta, et al, Phys. Rev. B 65 (5) 052411 (2002).

[30] S. Carretta, et al, Phys. Rev. B 67 (9) 094405 (2003).

[31] S. Carretta, et al, Phys. Rev. B 76, 024408 (2007).

[32] S. Carretta et al, Phys. Rev. Lett. 98, 167401 (2007).

[33] N. Cavadini et al, Phys. Rev. B 63, 172414 (2001).

[34] M. Cavallini et al, Nanoletters, 3, 1527 (2003).

[35] G. Chaboussant, et al, Phys. Rev. Lett. 80, 2713 (1998).

[36] G. Chaboussant, et al, Eur. Phys. J. B 6, 167 (1998).

[37] G. Chaboussant, et al, Phys. Rev. B 70104422 (2004).

[38] G. Chaboussant, et al, Europhys. Lett. 59291 (2002).

[39] G. Chaboussant, et al, Europhys. Lett. 66, 423 (2004).

[40] I. Chiorescu, et al, Phys. Rev. Lett. 84, 3454 (2000).

[41] I. Chiorescu, et al, Phys. Rev. B 67, 020402 (2003).

[42] J. Choi, et al, Phys. Rev. B. 68, 064412 (2003).

[43] G. Christou, Acc. Chem. Res. 22, 328 (1989).

[44] G. Christou, Polyhedron 24, 2065 (2005).

[45] M. Clemente-Leon, et al, Angew. Chem. Int. Ed. 372842 (1998).

[46] E. Coronado et A. Mingotaud, Advanced Materials, 11, 869 (1999).

[47] E. Dagotto, J. Riera and D. Scalapino, Phys. Rev. B. 45, 5744 (1992).

[48] E. Dagotto, Rep. Prog. Phys. 62, 1525 (1999).

[49] H. De Raedt et al, Phys. Rev. B. 70, 064401 (2004).

[50] J. R. Friedman, M. P. Sarachick, J. Tejada, et R. Ziolo, Phys. Rev. Lett. 76, 3830 (1996).

[51] A. Furrer et H.U. Güdel, J. Phys. C. Solid State Physics, 10, L191 (1977).

[52] A. Furrer and H.-U. Guedel, J. Mag. Mag. Mat. 14, 256 (1979).

[53] Y. Furukawa, et al, Phys. Rev. B. 62, 14246 (2000).

[54] J.S. Gardner, et al, Phys. Rev. Lett. 83, 211 (1999).

[55] D. Gatteschi, L. Pardi, A.L. Barra, A. Müller, et J. Döring, Nature, 354 (1991) 463.

[56] D. Gatteschi, Adv. Materials 6, 635 (1994).

[57] D. Gatteschi et L. Sorace, J. Solid. State. Chem. 159, 253 (2001).

[58] D. Gatteschi et R. Sessoli, Angewandte Chem. Int. Ed 42, 268 (2003).

[59] A.M. Gomes, M.A. Novak, R. Sessoli, A. Caneschi, and D. Gatteschi, Phys. Rev. B. 57, 5021 (1998).

[60] H.U. Güdel et A. Furrer, Mol. Phys. 33, 1335 (1977).

[61] M.A. Halcrow, J.S. Sun, J.C. Huffman et G. Christou, Inorg. Chem. 34, 4167 (1995).

[62] F.D.M. Haldane, Physical Review Letters 501153 (1983); F.D.M. Haldane, Physics Letters A 93, 464 (1983); F.D.M. Haldane, Journal Of Physics C-Solid State Physics 151309 (1982).

[63] J.S Helton et al, Phys. Rev. Lett. 98, 107204 (2007).

[64] S. Hill, et al, Phys. Rev. Lett. 80, 2453 (1998).

[65] S. Hill et al, Science 302, 1015 (2003).

[66] H. Kageyama, et al, Phys. Rev. Lett. 82, 3168 (1999).

[67] O. Kahn, Molecular Magnetism; VCH Publishers: New York (1993).

[68] O. Kahn, Acc. Chem. Res. 33, 647 (2000).

[69] G. Kamieniarz et al, J. Mag. Mag. Mat. 290-291, 970 (2005).

[70] K. Kim et al, Applied Physics Letters, 85, 3872 (2004).

[71] K. Kodama et al, Science 298, 395 (2002).

[72] K. Kojima et al, Phys. Rev. Lett. 74, 2812 (1995).

[73] A. Lascialfari et al, Phys. Rev. B 61, 6839 (2000).

[74] S.H. Lee et al, Nature 418, 856 (2002).

[75] M.N. Leuenberger et D. Loss, Nature 410789 (2001).

[76] T. Lis, Acta cristallog. Sec. B 36, 2042 (1980). 
[77] S.M. Lovesey, Theory of Thermal Neutron Scattering from Condensed Matter, (Clarendon Press Oxford) 1984.

[78] J.K. McCuster et al, J. Am. Chem. Soc. 113, 6114 (1991); ibid, 113, 3012 (1991).

[79] E.J.L. McInnes et al, Coord. Chem. Rev. 492577 (2005).

[80] N.D. Mermin et H. Wagner, Phys. Rev. Lett. 17, 1307 (1968).

[81] F. Mila, Eur. J. Phys. 21, 499 (2000).

[82] C.J. Milios et al, J. Am. Chem. Soc. 129, 8 (2007).

[83] C.J. Milios et al, J. Am. Chem. Soc. 1292754 (2007).

[84] I. Mirebeau et al, Phys. Rev. Lett. 83, 628 (1999).

[85] S. Miyahara and K. Ueda, Phys. Rev. Lett. 82, 3701 (1999).

[86] S. Miyahara et K. Ueda, Physica B 281, 661 (2000).

[87] A. Müller et J. Döring, Angew. Chem., Int. Ed. Engl. 27, 1721 (1988).

[88] A. Müller, F. Peters, M.T. Pope, et D. Gatteschi, Chem. Rev. 98, 239 (1998).

[89] Y. Murata et al, J. Phys. Soc. Jap. 67, 3014 (1998)

[90] S. Nakatsuji et al, Science 309, 5741 (2005).

[91] K.K. Nanda, L.K. Thompson, J.N. Bridson et K. Nag, J. Chem. Soc. Chem. Comm. 1337 (1992).

[92] K. Park, M.R. Pederson, and C.S. Hellberg, Phys. Rev. B 69, 014416 (2004).

[93] A.K. Powell et al, J. Am. Chem. Soc. 117, 2491 (1995).

[94] N.V. Prokofev, P.C.E. Stamp, J. Low. Temp. Phys. 104, 143 (1996).

[95] C. Raghu, I. Rudra, D. Sen, and S. Ramasesha, Phys. Rev. B 64, 064419 (2001).

[96] Pour une revue, voir A.P. Ramirez, in Handbook on Magnetic Materials 13, 423 (Elsevier Science, Amsterdam, 1979); S.T. Bramwell and M.J.P. Gingras, Science, 2941495 (2001); R. Moessner, Can. J. Phys. 79, 1283 (2001).

[97] A.P. Ramirez, Annual Review of Materials Science, 24, 453 (1994).

[98] N. Regnault N et al, Phys. Rev. B 66, 054409 (2002).

[99] Ch. Ruegg, N. Cavadini, A. Furrer, H.U. Gudel, K. Kramer, H. Mutka, A. Wildes, K. Habicht and P. Vorderwisch, Nature 423, 6935 (2003); Ch. Ruegg et al., Phys. Rev. Lett. 93, 257201 (2004); Ch. Ruegg et al, Phys. Rev. Lett. 93, 037207 (2004); Ch. Ruegg et al, Phys. Rev. Lett. 95, 267201 (2005); Ch. Ruegg et al, Phys. Rev. Lett. 100, 205701 (2008).

[100] D. Ruiz-Molina et al, Advanced Materials, 1542 (2003).

[101] S. Sachdev, Phys. Rev. B 45, 12377 (1992).

[102] Z. Salman et al, Nanoletters, 7, 1551 (2007).

[103] C. Sangregorio, T. Ohm, C. Paulsen, R. Sessoli, et D. Gatteschi, Phys. Rev. Lett. 78, 4645 (1997).

[104] P. Santini et al, Phys. Rev. B 71 (18) 184405 (2005).

[105] R. Sessoli et al, Nature 365, 141 (1993).

[106] R. Sessoli et al, J. Am. Chem. Soc. 115, 1804 (1993).

[107] B.S. Shastry and B. Sutherland, Physica B 108, 1069 (1981).

[108] A. Sieber et al, Angew. Chem. Int. Ed. 44, 4239 (2005).

[109] A. Sieber et al, Phys. Rev. B 70, 172413 (2004).

[110] G.L. Squires, "Introduction to the Theory of Thermal Neutron Scattering", Dover Publications; S.W. Lovesey, Theory of neutron scattering from condensed matter, Oxford University Press.

[111] Y. Suzuki, K. Takeda et K. Awaga, Phys. Rev. B 67, 132402 (2003).

[112] J. Tejada, et al, Nanotechnology 12, 181 (2001).

[113] L. Thomas et al, Nature 383, 145 (1996).

[114] D. Venegas-Yazigi et al, Dalton Trans., 2643 (2006).

[115] J. Villain, J. Phys. C 10, 1717 (1977).

[116] J. Villain, Z. Physik B 33, 31 (1979); J. Villain, R. Bideaux, J.P. Carton et R. Conte, J. Phys. (Paris) 41, 1263(1980).

[117] O. Waldmann et H. U. Güdel, Phys. Rev. B 72, 094422 (2005).

[118] O. Waldmann, C. Dobe, H. Mutka, A. Furrer et H.-U. Güdel, Phys. Rev. Lett. 95, 057202 (2005). 
[119] O. Waldmann, Phys. Rev. B 68, 174406 (2003).

[120] O. Waldmann, et al, Appl. Phys. Lett. 88, 042507 (2006).

[121] O. Waldmann et al, Phys. Rev. B 75, 174438 (2007).

[122] S. Wang et al, Inorg. Chem. 35, 7578 (1996).

[123] W. Wernsdorfer et R. Sessoli, Science 284 (5411) 133 (1999).

[124] S. Wessel, B. Normand, M. Sigrist et S. Haas, Phys. Rev. Lett. 86, 1086 (2001).

[125] S.R. White, Phys. Rev. B. 53, 52 (1996).

[126] K. Wieghardt, Angew. Chem., Int. Ed. Engl. 28, 1153 (1989); L. Que and A.E. True, Prog. Inorg. Chem. 38, 97 (1990); J.B. Vincent and G. Christou, AdV. Inorg. Chem. 33, 197 (1989); G. Christou, Acc. Chem. Res. 22, 328 (1989).

[127] V.K. Yachandra, K. Sauer and M.P. Klein, Chem. Rev. 96, 2927 (1996).

[128] T. Yamauchi et al, Phys. Rev. Lett. 83, 3729 (1999).

[129] Y.C Zhong et al, J. Appl. Phys. 85, 5636 (1999). 